\title{
Instrumentos de Medida de Actitudes hacia la Sexualidad: Una Revisión Bibliográfica Sistemática
}

\author{
Instruments for Measuring Attitudes toward Sexuality: A Systematic Review
}

\author{
Andrea Blanc Molina ${ }^{1}$ y Antonio J. Rojas Tejada ${ }^{1}$
}

\section{Resumen}

Las aproximaciones del estudio de las actitudes hacia la sexualidad se caracterizan por la abundancia de publicaciones basadas en distintos marcos conceptuales e instrumentos de medición. En este trabajo se realizó una revisión bibliográfica sistemática para identificar los instrumentos disponibles, con la finalidad de analizar sus objetivos, contenidos y principales características. Se utilizaron las bases de datos Proquest Psychology Journal y Scopus. En 372 documentos recuperados se identificaron 55 instrumentos diferentes que cumplían los criterios de inclusión establecidos, que permiten clasificarlos en tres tipos. Aunque los instrumentos hacen referencia a las actitudes hacia la sexualidad, no siempre comprenden contenidos similares. Esto es coherente con las definiciones amplias y poco delimitadas sobre la sexualidad y con las escasas definiciones del constructo actitudes hacia la sexualidad.

Palabras clave: actitudes, sexualidad, revisión sistemática

\begin{abstract}
The approaches to the study of attitudes toward sexuality are characterized by the abundance of publications based on different conceptual frameworks and by a plethora of measurement instruments. The aim of the study was to conduct a systematic literature review to identify and to present an overview of information (measurement objective, the content and the main properties) on instruments measuring the attitudes toward sexuality. A systematic literature search was undertaken in Proquest Psychology Journal and Scopus databases. Fifty five different instruments that met the inclusion criteria were identified in three hundred seventy-two retrieved documents. The instruments were classified in three types. Although the instruments refer to attitudes toward sexuality, not always refer to the same content. This is in line with the broad and slightly delimited definitions of sexuality and with the few explicit definitions of the construct attitudes toward sexuality.
\end{abstract}

Keywords: attitudes, sexuality, systematic review

Esta investigación se ha realizado gracias a la financiación del Ministerio de Educación, Cultura y Deporte para la Formación de Profesorado Universitario (Programa Estatal de Promoción del Talento y su Empleabilidad) adjudicado en concurso público en 2014.

\footnotetext{
${ }^{1}$ Grupo de Investigación HUM. 743. Estudios Psicosociales y Metodológicos. Facultad de Psicología, Universidad de Almería. 04120 Almería, España. UE. Correo: arojas@ual.es
} 


\section{Introducción}

Si hay una nota que caracteriza el campo de las actitudes hacia la sexualidad (actitudes sexuales), es su prolífica, diversa y dispersa producción tanto a nivel conceptual como en su medida. Sin embargo, esta abundancia de publicaciones no se corresponde con las exiguas definiciones explícitas del concepto de actitudes hacia la sexualidad que se pueden encontrar. Entre las escasas que aparecen en la literatura especializada, se encuentra la de López (2009), que la define como "una predisposición a opinar, sentir y actuar ante objetos sexuales (pornografía, por ejemplo), situaciones (el desnudo, por ejemplo), personas diferentes (homosexuales, por ejemplo), normas o costumbres sociales (el matrimonio, por ejemplo) y conductas sexuales (sexo oral, por ejemplo)" (p. 86).

A pesar de esa escasez, existe una gran diversidad de conceptos utilizados que hacen referencia al tema. Entre ellos, posiblemente se ha prestado mayor atención a los de permisividad sexual, conservadurismo-liberalismo sexual y erotofobia-erotofilia.

En la sociedad americana el concepto de permisividad sexual se ha asociado con el grado de afecto involucrado en la relación, siendo las personas más permisivas aquellas que aceptan con menos afecto una mayor variedad y tipologías de comportamientos sexuales (Reiss, 1964). Igualmente, la permisividad sexual se ha utilizado para referirse a las relaciones fuera del matrimonio, como las relaciones prematrimoniales $\mathrm{y}$ extramatrimoniales, $\mathrm{o}$ en general a las interacciones sexuales no maritales (Lottes \& Kuriloff, 1994). El constructo conservadurismoliberalismo sexual se ha considerado un continuo bipolar. En el polo del conservadurismo se situarían aquellas personas que sienten que la expresión de la sexualidad debe ser limitada y estrechamente regulada. En el polo del liberalismo, las personas que sienten que la expresión de la sexualidad deber ser abierta, libre y $\sin$ restricciones (Hudson, Murphy, \& Nurius, 1983). Por último, el constructo erotofobiaerotofilia se ha definido como la disposición aprendida a responder a estímulos sexuales a lo largo de un continuo bipolar de afecto $y$ evaluación, que se extiende desde un polo negativo (erotofobia) hasta un polo positivo (erotofilia) (Fisher, Byrne, White, \& Kelley, 1988). Las personas que se aproximan hacia el polo negativo responden con emociones y evaluaciones negativas ante los estímulos sexuales, y por tanto, los evitan más. Por el contrario, las personas más próximas al polo positivo muestran emociones y evaluaciones más favorables que los conducen a una mayor búsqueda de estímulos sexuales (Fisher et al., 1988).

En algunas investigaciones se han operacionalizado las actitudes hacia la sexualidad como el valor atribuido a diversos actos sexuales (Redfearn \& Laner, 2000), el grado de comodidad ante determinados comportamientos sexuales (McMillen, Helm, \& McBride, 2011), la aceptación de determinadas actividades sexuales de uno mismo y de los demás (Sprecher \& Hatfield, 1996), etc. Estas formas de operacionalizarlas muestran cómo se utilizan indistintamente en ocasiones diferentes las actitudes hacia la sexualidad y hacia los comportamientos sexuales. Asimismo, en una gran cantidad de investigaciones, por cuando se miden las actitudes hacia la sexualidad también se mide la realización de comportamientos sexuales (Lefkowitz, Gillen, Shearer, \& Boone, 2004; Luquis, Brelsford, \& Pérez, 2015; Luquis, Brelsford, \& Rojas-Guyler, 2012). De acuerdo con Marks \& Fraley (2005), las actitudes hacia los comportamientos sexuales pueden incluir creencias generales sobre las normas de una cultura, decisiones personales sobre la permisividad sexual y la percepción de la adecuación de ciertos comportamientos sexuales.

Por su parte, el concepto actitud ha sufrido una gran evolución a lo largo de la historia, y ha sido fuente de muchos debates. El modelo dominante sobre el concepto de actitud durante muchos años fue el Modelo de los tres componentes (Breckler, 1984; Brown, 1995; Eagly \& Chaiken, 1993; Zanna \& Rempel, 1988), que contempla una combinación peculiar de sentimientos, inclinaciones a la acción y creencias. Las manifestaciones evaluativas se dividen a su vez en tres clases: afectivas, conductuales y cognitivas. Actualmente se puede 
considerar, siguiendo a Fabrigar, MacDonald y Wegener (2005), que las actitudes pueden ser inferidas $\mathrm{y}$, al mismo tiempo estar influenciadas por las tres bases de información relevantes para la actitud (afectiva, conativa o conductual y cognitiva), asumidas tradicionalmente como componentes de la actitud (Brown, 1995; Eagly \& Chaiken, 1993). Esta concepción permite entender la actitud como una entidad separable de estas bases de información (Fabrigar et al., 2005). Cada una de ellas, separadas o conjuntamente, puede ser la fuente de información en la que se fundamente la actitud. Sin embargo, conocer las bases que influyen y a la vez son influidas por las actitudes no es equivalente a definir las actitudes. Cunningham, Zelazo, Packer y Van Bavel (2007) diferencian el concepto de actitud del concepto de evaluación. Por un lado, definen la actitud como un conjunto relativamente estable de representaciones almacenadas en la memoria sobre la valoración de un objeto. Por otro lado, definen la evaluación como un estado de procesamiento concreto que está influenciado por aquellos aspectos de la actitud que se encuentran activados en ese momento. De acuerdo con estos autores, la generación de evaluaciones se realiza de forma dinámica a través de la integración de las actitudes preexistentes y de la información adicional que proporciona el objeto actitudinal, el contexto y la relacionada con las metas (Cunningham et al., 2007).

Respecto a la sexualidad, a pesar de ser un campo muy investigado en las últimas décadas (Wells \& Twenge, 2005), las definiciones que se han proporcionado se caracterizan por ser muy amplias (engloban una gran variedad de cuestiones) y sin indicadores comportamentales claros (comportamientos bien definidos y delimitados) (López, 2009; OMS, 2006). La Organización Mundial de la Salud (OMS, 2006) define la sexualidad como un aspecto central del ser humano a lo largo de toda la vida, que abarca el sexo, las identidades y roles de género, el erotismo, el placer, la intimidad, la reproducción y la orientación sexual (OMS, 2006).

La diversidad de términos utilizados en la investigación sobre las actitudes hacia la sexualidad, las diferentes definiciones del concepto de actitud en que se basan así como las definiciones amplias y poco delimitadas de sexualidad, no han supuesto obstáculo alguno para los trabajos sobre la medición de actitudes hacia la sexualidad. Lejos de ello, en la literatura aparecen abundantes instrumentos de medida

El objetivo del presente estudio ha sido realizar una búsqueda bibliográfica sistemática de los documentos que contenían instrumentos de medida de actitudes hacia la sexualidad, con la finalidad de mostrar cuáles son los objetivos explícitos de medición para los que se han construido, las dimensiones o contenidos incluidos, y características tales como población objetivo, contexto de aplicación, número y formato de ítems, entre otros.

\section{Método}

\section{Estrategia de búsqueda}

Se ha llevado a cabo una búsqueda bibliográfica sistemática de los documentos en los que se mencionan instrumentos del tipo considerado en las bases de datos Proquest Psychology Journals y Scopus. Lo criterios de búsqueda utilizados han sido: documentos restringidos a revistas científicas y profesionales (fuentes primarias) escritos en español y en inglés y términos limitados al campo del título y sin restricción temporal, que corresponden a: "attitud toward sexuality", "attitud to sexuality", "sexual attitud", "actitud hacia la sexualidad" y "actitud sexual".

\section{Criterios de inclusión}

Para la elección de los documentos se han considerado tres criterios de inclusión, de acuerdo a la siguiente propuesta:

1. Documentos en los que aparecen instrumentos que miden únicamente actitudes sexuales o actitudes hacia la sexualidad en general. Se descartaron todos los que incluyen instrumentos que miden actitudes hacia aspectos muy específicos de la sexualidad como, por ejemplo, homosexualidad (Lozano \& Díaz-Loving, 2010), autoesquema sexual (Martínez, Reyes, \& Paredes, 2010), etc.

2. Documentos en los que aparecen alguna escala o subescala que evalúan actitudes sexuales $o$ actitudes hacia la sexualidad. Se han 
considerado aquellos instrumentos que, además de medir actitudes sexuales, miden otros constructos, por ejemplo conocimiento sexual.

3. Documentos en los que aparecen instrumentos que se han diseñado únicamente para un estudio en concreto, pero que en dicho estudio consta explícitamente el instrumento o los ítems del mismo en su totalidad. Se descartan todos aquellos instrumentos construidos ad hoc para un solo estudio y no reflejados en la publicación.

\section{Procedimiento}

La revisión se llevó a cabo en enero de 2015, de forma independiente por los dos investigadores. Recuperados todos los documentos tras realizar la búsqueda, se examinaron aquellos que cumplían con los criterios de inclusión. Una vez seleccionados se analizaron en profundidad. Debido a la insuficiencia de información sobre los instrumentos utilizados en distinto estudios, se recurrió a fuentes secundarias y terciarias para completarla, tales como The Handbook of Sexuality-Related Measures (Fisher, Davis, Yarber, \& Davis, 2011) y Sex and Gender Issues: A Handbook of Test and Measures (Beere, 1990).

\section{Codificación de los resultados}

De las fuentes consultadas se obtuvo la siguiente información: nombre completo del instrumento de medida y sus revisiones, autor/es, primer año de aparición, número de ítems, formato de respuesta, escalas o subescalas que lo integran, contenido, dimensiones o factores explicitados, estimación de la fiabilidad y población objetivo para el que fue construido.

\section{Resultados}

La búsqueda dio lugar a 372 artículos, en los que se encontraron 55 en los que se mencionaban instrumentos que cumplían con los criterios de inclusión. Cabe destacar que 30 de los artículos recuperados hacían referencia a dos estudios muy amplios: The British National Survey of Sexual Attitudes and Lifestyle (21 artículos) y The Global Study of Sexual Attitude and Behavior (9 artículos). Los instrumentos encontrados se han agrupado en tres categorías:
1. Instrumentos que miden únicamente actitudes hacia la sexualidad. Los 30 instrumentos de este tipo se presentan en orden cronológico de construcción, excepto en los casos de la Premarital Sexual Permissiveness Scale (PSPS) (Reiss, 1989; Sprecher, McKinney, Walsh, \& Anderson, 1988) y la Brief Sexual Attitude Scale (BSAS) (Hendrick, Hendrick, \& Reich, 2006) en los que se muestran las revisiones a continuación de la versión original (Reiss, 1964; Hendrick, Hendrick, Slapion-Foote, \& Foote, 1985) (Tabla 1).

En relación con el contenido/dimensiones/ escalas/componentes/factores explicitados en los instrumentos, existe una gran variedad a pesar de que todos hacen referencia a la medida de las actitudes hacia la sexualidad. Por ejemplo, la PSPS (Reiss, 1964) se centra en las relaciones prematrimoniales y la Attitude Toward Marital Exclusivity (Weis \& Felton, 1987) en las relaciones extramatrimoniales, siendo ambas medidas, según los autores, medidas unidimensionales de las actitudes hacia la sexualidad. La Sexual Attitude Scale (SAS) en cambio es una medida multidimensional, ya que examina otros factores como la responsabilidad sexual, la comunión sexual, la instrumentalidad sexual, etc. (Hendrick et al., 1985).

De todos los instrumentos, el Trueblood Sexual Attitudes Questionnaire (TSAQ) es el único que mide las actitudes personales hacia la sexualidad (40 ítems) y las actitudes hacia la sexualidad de las personas en general (40 ítems), utilizando dos escalas con el mismo contenido en los ítems (Trueblood, Hannon, \& Hall, 1998). No obstante, a pesar de incluirlos dentro de este tipo, el contenido de algunos instrumentos se centra diferencialmente en mujeres y hombres y también miden los dobles estándares sexuales o doble moral sexual. Por ejemplo, la PSPS puede aplicarse, cambiando el target, para observar si hay diferencias en la aceptación de los comportamientos de hombres y de mujeres (Reiss, 1964). La Sexual Double Standards (Muehlenhard \& Quackenbush, 1996) contiene ítems que hacen referencia a las mujeres y otros a los hombres, y el cuestionario Attitude to Sexuality (Cuskelly \& Gilmore, 2007) tiene dos formas, una donde se 
mide la actitud hacia la expresión de la sexualidad de las mujeres y otra respecto a la de los hombres.

2. Instrumentos con alguna escala o subescala que mide actitudes hacia la sexualidad. Se identificaron 20 instrumentos. También se presentan según el orden cronológico de publicación, excepto el SKAT-A (Lief, Fullard, \& Devlin, 1990) que es una adaptación del SKAT (Lief \& Reed, 1972) para jóvenes y se muestra a continuación del mismo (Tabla 2).

3. Instrumentos construidos para medir las actitudes hacia la sexualidad de las personas hacia colectivos específicos. Por último, se identificaron 5 instrumentos con esas características, que también se presentan en orden cronológico. Dos de ellos se centran en la actitudes sexuales hacia las personas mayores (ASKAS y Escala de Actitudes hacia la Sexualidad en el Envejecido) y tres en la actitudes sexuales hacia las personas con discapacidad intelectual o del aprendizaje (Questionnaire about attitudes of staff in services for people with intellectual disabilities, ASQ-ID y GSAQ-LD). Cabe señalar que el ASKAS mide conocimiento sexual, pudiéndose agrupar también en la segunda categoría y el GSAQ-LD las actitudes hacia las personas sin discapacidad, pudiéndose agrupar en la primera (Tabla 3).

\section{Características generales de todos los instrumentos analizados}

La mayoría de los instrumentos posee un formato de respuesta elegida tipo Likert. La población, el ámbito, el objetivo, los contenidos y las propiedades psicométricas de cada uno de ellos son diversos. En el caso de la población y el objetivo, los instrumentos se han aplicado a la población general (por ejemplo el SOI), y a poblaciones muy específicas, como pueden ser los delincuentes que han cometido agresiones sexuales (Sex Inventory). El ámbito de aplicación considerado ha sido variado, como por ejemplo centros de enseñanza (TSAQ), centros penitenciarios (Index Liberal Sexual Attitude) y centros sanitarios (SABS), entre otros.

Considerando el contenido/dimensiones/ escalas/componentes/factores de los instrumentos, se encuentra que cuando se miden las actitudes hacia la sexualidad, los autores se refieren a una diversidad de conceptos: comportamientos sexuales prematrimoniales (PSPS), comportamientos sexuales extramatrimoniales (Attitude Toward Marital Exclusivity Scale) comportamientos homosexuales (SOS), comportamientos heterosexuales (SOS), doble moral sexual (SDSS), sexo oral y sexo durante la menstruación (Sexual Conservatism Scale), masturbación (Valois Sexual Attitude Scale), fantasías sexuales (Sexuality Scale), prostitución (ATSS), pornografía (Sexual Attitude Questionnaire), aborto (Attitude Toward Sexuality Inventory), infecciones de transmisión sexual (ATSS), métodos anticonceptivos (ASQ-GP), roles de género (MAV), educación sexual (ASQID), violación sexual (SKAT-A), etc. En algunas escalas incluso se mide la actitud hacia los comportamientos sexuales según cierto grado de afecto o estado relacional (PSPS). También son objetos de medición los pensamientos sobre la sexualidad (Sexuality Scale), la culpa sexual (The Mosher Forced Choice Sex Guilt Scale), las preocupaciones sexuales (SAIQ), la comunicación sexual (Valois Sexual Attitude Scale) y la comodidad sexual (CCAS). Por último, también se observa cómo se evalúa la importancia del orgasmo (Sexual Attitude Questions) y la excitación sexual (Sexuality Scale).

En cuanto a las propiedades psicométricas, la fiabilidad de las puntuaciones se ha estimado principalmente, pero no en todos los casos, mediante el coeficiente $\alpha$ de Cronbach y la correlación test-retest (coeficiente de estabilidad).

\section{Discusión}

El objetivo del estudio ha sido realizar una búsqueda bibliográfica sistemática de documentos que hacían referencia a instrumentos que se han utilizado para medir lo que en la literatura se denomina actitudes hacia la sexualidad, con la finalidad de describir cuál es el objetivo de medición, qué se está midiendo (contenidos/dimensiones), y cómo se está midiendo. Los resultados muestran una gran variedad de instrumentos con diferentes características, que se han clasificado en los tres tipos incluidos. 
En la primera categoría (instrumentos que miden únicamente actitudes hacia la sexualidad), el primero que se construyó fue la PSPS, que se reduce a medir las actitudes hacia comportamientos sexuales prematrimoniales (Reiss, 1964). The Sexual and Premarital Attitude Inventory (Schofield, 1965) también se centra en interacciones sexuales antes del matrimonio, pero introduce otros contenidos como la homosexualidad. Con el paso del tiempo, se observa cómo la medición de actitudes hacia la sexualidad no se reduce a comportamientos sexuales prematrimoniales y cuando lo hace se eliminan los menos permisivos. La última versión de la PSPS (Reiss, 1989) ya no incluye la actitud hacia comportamientos sexuales como los besos y el petting, posiblemente por la aceptación de las relaciones prematrimoniales de la mayoría de los jóvenes (Reiss, 2006).

Los instrumentos que se han construido pocos años más tarde, como el Inventory of Attitude to Sex o el Sex Inventory (Eysenck, 1970; Thorne, 1966), se han focalizado en colectivos más específicos como los agresores sexuales, y han medido la actitud hacia determinados comportamientos sexuales, considerando algunos tipos de conductas sexuales poco adaptativas e inmorales, causantes incluso de conflicto o neurosis (Eysenck, 1970; Thorne, 1966). Con el SAS (Hendrick et al., 1985) aparece un instrumento explícitamente multidimensional de actitudes hacia la sexualidad.

Esta evolución de los instrumentos de medida es coherente con los cambios que se van produciendo en las sociedades en relación con las actitudes hacia la sexualidad (Croake \& James, 1973; Glenn \& Weaver, 1979; Wells \& Twenge, 2005).

En la segunda categoría (instrumentos encontrados con alguna escala o subescala que mide actitudes hacia la sexualidad), se observan diferencias en el contenido de los instrumentos dependiendo de la población para la que se han construido. Por ejemplo, en el Sexual Attitude and Information Questionnaire (SAIQ; Brockway et al., 1978) se miden preocupaciones sexuales, en el Sexual Dysfunctional Beliefs Questionnaire (SDBQ; Nobre, Pinto-Gouveia, \& Gomes, 2003) creencias sexuales disfuncionales y en el Self-
Image Questionnaire (Offer, 1972) la autopercepción del atractivo hacia el otro género. La construcción de este tipo de instrumentos muestra cómo las actitudes hacia la sexualidad son relevantes en las alteraciones orgánicas, en las disfunciones sexuales, en trastornos de la imagen corporal, etc.

Por último, en la tercera categoría, donde se han agrupado los instrumentos que miden las actitudes sexuales hacia colectivos específicos (personas mayores y persona con discapacidad intelectual) observamos la aparición de nuevos factores vinculados con la crianza, los derechos sexuales o la discriminación. En el caso de los instrumentos que miden las actitudes hacia la sexualidad de las personas con discapacidad intelectual, el primero publicado aparece en el año 2000 , fruto de los cambios que se han producido en la sociedad respecto a la consideración de ese colectivo poblacional.

En su conjunto, el análisis de los instrumentos de medida muestra una gran diversidad de objetivos, contenidos/dimensiones/componentes/ escalas/factores explicitados, así como de las poblaciones a las que van dirigidos, relacionados con la existencia de numerosas definiciones diferentes sobre la sexualidad.

En resumen, tras la revisión y análisis de los instrumentos de medida, se han encontrado instrumentos heterogéneos para medir actitudes hacia la sexualidad. La panorámica cronológica permite, además, observar un cambio en tales actitudes, que han ido modificado los comportamientos sexuales a los que se hace referencia y el surgimiento de nuevos instrumentos destinados a atender las necesidades sexuales de personas discapacitadas y mayores. Esta evolución refleja el cambio de las actitudes de la población hacia la sexualidad y la evolución del concepto de sexualidad (Gray, 2013), que resulta cada vez más amplio.

A pesar de mencionar los conceptos de actitudes hacia la sexualidad, la diversidad de instrumentos hallados revela que no siempre se está midiendo lo mismo. Los resultados del análisis realizado son coherentes con las definiciones amplias y poco delimitadas de sexualidad, con las escasas definiciones explícitas del constructo y con los diferentes conceptos que 
se utilizan cuando se miden las actitudes hacia la sexualidad (permisividad sexual, conservadurismo-liberalismo sexual, erotofobiaerotofilia). Dentro de este corpus heterogéneo y difícil de delimitar, no solo resulta necesario el análisis de instrumentos de medida, sino que, inevitablemente se debería contar con una definición más precisa de las actitudes hacia la sexualidad que pueda ser mayoritariamente consensuada por los especialistas en el tema.

\section{Tablas}

Tabla 1. Revisión de instrumentos que miden únicamente actitudes hacia la sexualidad

\section{Título $\quad \mathbf{N}^{\mathbf{0}}$ ítems \\ Autores y año de la primera referencia \\ Contenido/Dimensiones/Componentes/ Escalas/Factores y Fiabilidad estimada respuesta}

\section{Premarital Sexual Permissiveness Scale (PSPS)}

Reiss (1964)

\section{Premarital Sexual} Permissiveness Scale (PSPS) (revisión de la PSPS de Reiss, 1964)

Sprecher, McKinney, Walsh, \& Anderson (1988)

3. The Short Form of the Reiss Male and Female Premarital Sexual Permissiveness Scale (versión corta de la PSPS) Reiss (1989)

\section{The Sexual and} Premarital Attitude Inventory

Schofield (1965)

\section{Sex Inventory}

Thorne (1966)

\section{Measure of Sexual} Attitude

Marks \& Sartorius (1968)

7. Sexual LiberalismConservatism Scale Athanasiou \& Shaver (1969)
12 ítems

Guttman con 6 opciones: TA-TD.

\section{5 ítems}

Likert con 6 opciones: TATD.

\section{4 ítems}

Guttman con 6 opciones: TA-TD.

17 ítems

Likert con 3 opciones: de acuerdo, neutral y en desacuerdo.

200 ítems

2 opciones de respuesta: síno.

26 adjetivos

(13 pares)

Diferencial Semántico.

16 ítems

Likert con 6 opciones.
Componentes sexuales: besos, petting y coito. Niveles de afecto: no afecto, fuerte afecto, enamorado y comprometido.

Fiabilidad: $\mathrm{CR}>.90 ; \mathrm{CE}>.65$.

Componentes sexuales: caricias en los genitales, coito y sexo oral.

Niveles de afecto: primera cita, citas casuales (menos de un mes), citas serias (casi un año), pre-comprometido (compromiso informal para el matrimonio) y comprometido (comprometido formalmente para casarse). Fiabilidad: $\alpha=.94$.

Componente sexual: coito.

Niveles de afecto: sin mucho afecto, moderado afecto, fuerte afecto y amor.

Fiabilidad: $\alpha=.89$.

Contenido: sexo antes del matrimonio de hombres y mujeres, matrimonio, supervisión parental, educación sexual, familia y divorcio y homosexualidad.

Escalas: deseo e interés sexual, inadaptación sexual y frustración, conflicto neurótico asociado con el sexo, represión sexual, pérdida de control sexual, homosexualidad, confianza en los roles de género y actitudes sexuales sociópatas.

Factores: evaluación general, evaluación sexual y ansiedad.

Contenido: homosexualidad, prostitución, educación sexual, control de la natalidad, doble moral sexual, etc.

Fiabilidad: $\alpha=.84$.

\section{Objetivo y población explicitados}

Actitudes hacia la aceptabilidad de comportamientos sexuales premaritales en población general no casada, según diferentes grados de afecto (Estados Unidos).

Actitudes hacia la aceptabilidad de comportamientos sexuales premaritales en población general no casada, según diferentes niveles de desarrollo relacional (Estados Unidos).

Actitudes hacia la aceptabilidad del sexo premarital en diferentes grados de afecto en población general no casada (Estados Unidos).

Actitud hacia el sexo y hacia la interacción de hombres y mujeres antes del matrimonio en población iraní (Estados Unidos).

Desajuste sexual con respecto a los comportamientos, actitudes y autopercepciones de delincuentes que cometen agresiones sexuales.

Actitudes sexuales en pacientes en tratamiento por desviación sexual para evaluar su progreso.

Posturas liberales y conservadoras hacia la sexualidad en estudiantes y sus padres. 
Tabla 1 (cont.).Revisión de instrumentos que miden únicamente actitudes hacia la sexualidad

\begin{tabular}{|c|c|c|c|}
\hline $\begin{array}{l}\text { Título } \\
\text { Autores y año de la } \\
\text { primera referencia }\end{array}$ & $\begin{array}{l}\mathrm{N}^{0} \text { ítems } \\
\text { Formato de } \\
\text { respuesta }\end{array}$ & $\begin{array}{l}\text { Contenido/Dimensiones/Componentes/ } \\
\text { Escalas/Factores y Fiabilidad estimada }\end{array}$ & $\begin{array}{l}\text { Objetivo y población } \\
\text { explicitados }\end{array}$ \\
\hline
\end{tabular}

98 ítems

(1970)

8. Inventory of Attitude to

Sex (adaptado del

Inventario de Thorne y

revisado en 1976)

Eysenck (1970)

9. The Dearth Cassell

Attitude Inventory

Dearth \& Cassell (1976)

10. Sexual Opinion Survey (SOS) (versión corta por Smeph en 1979)

White, Fisher, Byrne, \& Kingma (1977)

\section{Sexual Conservatism Scale}

Burt (1980)

12. Valois Sexual Attitude Scale

Valois (1980)

\section{Sexuality Scale \\ Irving \& Thissen \\ (1983)}

14. Sexual Attitude Scale (SAS)

Hudson, Murphy, \& Nurius (1983)

(1976) no puedo decir y

falso/no.

18 ítems opciones.

21 ítems

5 ítems

(1979)

10 ítems opciones: TA-TD.

46 ítems opciones: TA-TD.

21 ítems opciones: extremaextrema.

25 ítems opciones: TA-TD.
158 ítems

3 opciones: verdadero/sí,

Contenido: control de la natalidad, religión, homosexualidad, sexo en grupo,

Likert con 5

Likert con 7 Factores: exhibición de la sexualidad, opciones: variedad sexual y homoerotismo.

TA-TD. Fiabilidad: $\alpha$ (hombres) $=.86 ; \alpha$ (mujeres) $=.82 ; \mathrm{r}$ $($ dos semanas $)=.84 ; \mathrm{r}($ dos meses $)=.85$.

Likert con 7

Likert con 5

Likert con 5 comodidad

incomodidad

Likert con 5

Escalas: permisividad, satisfacción sexual, sexo neurótico, sexo impersonal, pornografía, timidez sexual, puritanismo, excitación sexual, dominancia-sumisión, repugnancia sexual, sexo físico y sexo agresivo. enfermedades venéreas, cuerpo masculino, aborto, discusión sexual, educación sexual, comportamiento sexual adulto, deseo sexual, terapia sexual, relaciones sexuales, masturbación, consentimiento adulto, cohabitación, infidelidad y pornografía.

Estímulos sexuales: comportamiento autosexual, comportamiento homosexual, comportamiento heterosexual, fantasías sexuales y estímulos visuales sexuales.

Subescalas: estereotipos sexuales, masturbación, relaciones prematrimoniales, homosexualidad, comunicación sexual, matrimonio universidad, aborto, sexo oral y control de la natalidad.

Fiabilidad: $\alpha$ en cada subescala oscila entre .66 y .92 .

Factores y fiabilidad: pensamiento sobre sexo y ser una persona sexual $(\alpha=.86)$, frecuencia de pensamientos sexuales, fantasías sexuales y excitación $(\alpha=.82)$, importancia de la placer de participar en la actividad sexual y ser una persona sexual $(\alpha=.87)$.

Actitudes sexuales: conservadoras (expresión de la sexualidad limitada y estrechamente regulada) y liberales (expresión de la sexualidad abierta, libre y sin restricciones).
Respuestas afectivas y evaluativas a estímulos sexuales (constructo erotofobia-erotofilia) en una población amplia.

Cambio de actitudes después de un curso de sexualidad humana en estudiantes.

Restricciones sobre la idoneidad de las parejas sexuales, actos sexuales, condiciones o circunstancias bajo las que debe ocurrir sexo, en hombres y mujeres, hombres condenados por violación, pederastas, otros delincuentes, etc. sexualidad en la vida de uno mismo $(\alpha=.86)$, Fiabilidad: $\alpha=.94$.
Actitudes hacia nueve temas sexuales en profesionales sanitarios.

Confort intrapersonal con la sexualidad en mujeres con trastornos de alimentación.

Actitudes liberales versus conservadoras hacia la expresión de la sexualidad humana, principalmente en estudiantes. 
Tabla 1 (cont.). Revisión de instrumentos que miden únicamente actitudes hacia la sexualidad

\begin{tabular}{|c|c|c|c|}
\hline $\begin{array}{l}\text { Título } \\
\text { Autores y año de la } \\
\text { primera referencia }\end{array}$ & $\begin{array}{l}\mathrm{N}^{0} \text { ítems } \\
\text { Formato de } \\
\text { respuesta }\end{array}$ & $\begin{array}{l}\text { Contenido/Dimensiones/Componentes/ } \\
\text { Escalas/Factores y Fiabilidad estimada }\end{array}$ & $\begin{array}{l}\text { Objetivo y población } \\
\text { explicitados }\end{array}$ \\
\hline
\end{tabular}

15. Sexual Attitude Scale (SAS)

Hendrick, Hendrick, Slapion-Foote, \& Foote (1985)
$102(1985)$

58 (1985)

43 (1987)

Likert con 5 opciones: TA-TD.
Factores, fiabilidad y contenido (43 ítems): permisividad sexual $(\alpha=.93)$ : actitud abierta hacia el sexo y sexo casual; prácticas sexuales $(\alpha=.82)$ : responsabilidad (métodos anticonceptivos) y tolerancia (masturbación); comunión sexual $(\alpha=.74)$ : sexo como una experiencia ideal e instrumentalidad sexual ( $\alpha$ $=.78$ ): sexo como natural, biológico y auto orientado a aspectos de la vida.

16. Brief Sexual Attitude Scale (BSAS) (revisión del SAS de Hendrick et al., 1987)

Hendrick, Hendrick, \& Reich (2006)

\section{Attitudes Toward} Sexuality Scale (ATSS)

Fisher (1986)

\section{Attitude Toward Marital Exclusivity Scale}

Weis \& Felton (1987)

\section{Attitude Toward} Sexuality Inventory (revisión en 1995)

Patton \& Mannison (1993)
23 ítems

Likert con 5 opciones: TA-TD.

14 ítems

Likert con 5 opciones:

TA-TD.

7 presentados con viñetas

Likert con 5 opciones: aceptación total-rechazo total.

72 ítems

(1993)

40 ítems (1995) opciones:

TA-TD.
Likert con 6
Factores y fiabilidad: permisividad sexual ( $\alpha$ $=.85)$, control de la natalidad $(\alpha=.88)$, comunión sexual $(\alpha=.82)$ e instrumentalidad sexual $(\alpha=.80)$.

Contenido: nudismo, aborto, contracepción, sexo prematrimonial, pornografía, prostitución, homosexualidad y enfermedades de transmisión sexual.

Factores: legalidad-moralidad, maneras de expresar la sexualidad y derechos individuales.

Fiabilidad: $\alpha$ (12-14 años) $=.76 ; \alpha(15-17$ años $)=.65 ; \alpha(18-20$ años $)=.80 ; \alpha$ (padres $)$ $=.84$.

Contenido: relaciones extramatrimoniales. Fiabilidad: $\alpha=.84$.

Contenido: contracepción, masturbación, sexualidad a través de la vida, roles de género, relaciones homosexuales, aborto, enfermedades de transmisión sexual, abuso sexual infantil, violación y educación sexual.

Factores y fiabilidad (1995): asalto y coerción sexual $(\alpha=.85)$, cuestiones sexuales $(\alpha=.79)$ y roles de género $(\alpha=.68)$.
Actitudes sexuales de una fuerte dependencia de la permisividad sexual a un enfoque más integral y multidimensional en población general.

Actitudes sexuales de adolescentes entre 12 y 20 años y sus padres.

Actitudes hacia las relaciones sexuales extramatrimoniales tras un curso de sexualidad humana en estudiantes.

Cambio de actitudes después de un curso de sexualidad humana en estudiantes. 
Tabla 1 (cont.). Revisión de instrumentos que miden únicamente actitudes hacia la sexualidad

\begin{tabular}{|c|c|c|c|}
\hline $\begin{array}{l}\text { Título } \\
\text { Autores y año de la } \\
\text { primera referencia }\end{array}$ & $\begin{array}{l}\mathbf{N}^{0} \text { ítems } \\
\text { Formato de } \\
\text { respuesta }\end{array}$ & $\begin{array}{l}\text { Contenido/Dimensiones/Componentes/ } \\
\text { Escalas/Factores y Fiabilidad estimada }\end{array}$ & $\begin{array}{l}\text { Objetivo y población } \\
\text { explicitados }\end{array}$ \\
\hline $\begin{array}{l}\text { 20. Survey of Sexual } \\
\text { Attitude } \\
\text { Calfin, Carroll, \& Schmidt } \\
\text { (1993) }\end{array}$ & $\begin{array}{l}18 \text { ítems } \\
2 \text { opciones: } \\
\text { de acuerdo-en } \\
\text { desacuerdo. }\end{array}$ & & $\begin{array}{l}\text { Actitudes sexuales premaritales } \\
\text { tras visualizar cintas de vídeo } \\
\text { musicales con contenido } \\
\text { romántico y con contenido } \\
\text { erótico en estudiantes. }\end{array}$ \\
\hline $\begin{array}{l}\text { 21. The Sexual } \\
\text { Socialization Instrument } \\
\text { (SSI) } \\
\text { Lottes \& Kuriloff (1994) }\end{array}$ & $\begin{array}{l}20 \text { ítems } \\
\text { Likert con } 5 \\
\text { opciones: } \\
\text { TA-TD. }\end{array}$ & $\begin{array}{l}\text { Escalas y fiabilidad: Peer Sexual Socialization } \\
\text { Scale }(12 \text { ítems })(\alpha=.85) \text { y Parental Sexual } \\
\text { Socialization Scale ( } 8 \text { ítems })(\alpha=.78) \text {. }\end{array}$ & $\begin{array}{l}\text { Influencias sexuales permisivas } \\
\text { de padres y grupo de iguales en } \\
\text { adolescentes y adultos jóvenes. }\end{array}$ \\
\hline $\begin{array}{l}\text { 22. Attitude Toward } \\
\text { Sexuality } \\
\text { Huerta, Mena, Malacara, \& } \\
\text { Díaz De León (1995) }\end{array}$ & $\begin{array}{l}13 \text { ítems } \\
\text { Likert con } 11 \\
\text { opciones. }\end{array}$ & $\begin{array}{l}\text { Contenido: roles de la mujer como ama de } \\
\text { casa, atracción física y actividad sexual } \\
\text { (iniciativa de la mujer en la actividad sexual y } \\
\text { actividad sexual durante la menopausia). }\end{array}$ & $\begin{array}{l}\text { Opinión sobre temas sexuales en } \\
\text { mujeres perimenopaúsicas (etapa } \\
\text { previa a la menopausia). }\end{array}$ \\
\hline $\begin{array}{l}\text { Muehlenhard \& } \\
\text { Quackenbush (1996) }\end{array}$ & $\begin{array}{l}26 \text { ítems } \\
\text { Likert con } 4 \\
\text { opciones: } \\
\text { TA-TD. }\end{array}$ & $\begin{array}{l}\text { Contenido: sexo prematrimonial, múltiples } \\
\text { parejas, sexo a edad joven y nuevas o } \\
\text { relaciones sin compromiso. } \\
\text { Fiabilidad: } \alpha \text { (hombres) }=.76 ; \alpha \text { (mujeres) }= \\
.73 \text {. }\end{array}$ & $\begin{array}{l}\text { Adherencia a la doble moral } \\
\text { sexual tradicional de la población } \\
\text { general. }\end{array}$ \\
\hline $\begin{array}{l}\text { 24. The Trueblood Sexual } \\
\text { Attitudes Questionnaire } \\
\text { (TSAQ) (revisión en 1999) } \\
\text { Trueblood, Hannon, \& Hall } \\
\text { (1998) }\end{array}$ & $\begin{array}{c}90 \text { ítems } \\
(1998) \\
80 \text { ítems } \\
(1999): 40 \\
\text { personales y } \\
40 \text { hacia los } \\
\text { demás. } \\
\text { Likert con } 9 \\
\text { opciones: } \\
\text { completamente } \\
\text { de acuerdo- } \\
\text { completamente } \\
\text { en desacuerdo. }\end{array}$ & $\begin{array}{l}\text { Subescalas y fiabilidad (personales y hacia los } \\
\text { demás, respectivamente): autoerotismo }(\alpha= \\
.88 \text { y } .91) \text {, homosexualidad }(\alpha=.91 \text { y } .98) \text {, } \\
\text { heterosexualidad }(\alpha=.68 \text { y } .56) \text {, variaciones } \\
\text { sexuales }(\alpha=.78 \text { y } .88) \text { y sexo comercial }(\alpha \\
=.76 \text { y } .81) .\end{array}$ & $\begin{array}{l}\text { Cambio de actitudes con respecto } \\
\text { a temas comunes relacionados } \\
\text { con el comportamiento sexual } \\
\text { tras un curso de sexualidad } \\
\text { humana en estudiantes. }\end{array}$ \\
\hline $\begin{array}{l}\text { 25. Attitudes Toward } \\
\text { Dating and Relationships } \\
\text { Measure } \\
\text { Ward \& Rivadeneyra } \\
\text { (1999) }\end{array}$ & $\begin{array}{l}33 \text { ítems } \\
\text { Likert con } 6 \\
\text { opciones: } \\
\text { TA-TD. }\end{array}$ & $\begin{array}{l}\text { Contenido: noviazgo, roles sexuales y } \\
\text { relaciones románticas. } \\
\text { Subescalas y fiabilidad: orientación recreativa } \\
\text { hacia la sexualidad }(\alpha=.84) \text {, orientación de } \\
\text { procreación o tradicional hacia la sexualidad } \\
(\alpha=.75) \text { y orientación relacional hacia la } \\
\text { sexualidad }(\alpha<.70) \text {. }\end{array}$ & $\begin{array}{l}\text { Actitudes hacia el noviazgo, los } \\
\text { roles sexuales y las relaciones } \\
\text { románticas en adolescentes, para } \\
\text { observar en qué medida los } \\
\text { medios de comunicación } \\
\text { influyen. }\end{array}$ \\
\hline $\begin{array}{l}\text { 26. Sexual Attitude } \\
\text { Questions } \\
\text { Kupek (2001) }\end{array}$ & $\begin{array}{l}8 \text { ítems } \\
\text { Likert con } 6 \\
\text { opciones: } \\
\text { siempre es un } \\
\text { error-en absoluto } \\
\text { es un error. }\end{array}$ & $\begin{array}{l}\text { Factores: permisividad sexual, aceptación de } \\
\text { la homosexualidad e importancia del orgasmo } \\
\text { para el sexo. }\end{array}$ & $\begin{array}{l}\text { Opinión hacia las relaciones } \\
\text { sexuales y otros temas sexuales } \\
\text { en la población general (Gran } \\
\text { Bretaña). }\end{array}$ \\
\hline $\begin{array}{l}\text { 27. Comfort With Personal } \\
\text { Sexuality } \\
\text { Rempel \& Baumgartner } \\
\text { (2003) }\end{array}$ & $\begin{array}{l}10 \text { ítems } \\
\text { Likert con } 7 \\
\text { opciones: } \\
\text { TA-TD. }\end{array}$ & $\begin{array}{l}\text { Contenido: deseo sexual y expresión de la } \\
\text { sexualidad como un aspecto natural de la vida. } \\
\text { Fiabilidad: } \alpha=.81 \text {. }\end{array}$ & $\begin{array}{l}\text { Grado en que las mujeres se } \\
\text { sienten cómodas, abiertas y } \\
\text { aceptan la sexualidad. }\end{array}$ \\
\hline
\end{tabular}


Tabla 1 (cont.). Revisión de instrumentos que miden únicamente actitudes hacia la sexualidad

\section{Título}

Autores y año de la primera referencia

28. Attitudes to Sexuality

Questionnaire (ASQ-GP)

Cuskelly \& Gilmore (2007)

\section{Sexual Attitude}

Questionnaire

Kang (2007)

30. Escala de Actitud hacia la Sexualidad

Lima-Serrano, Sáez-

Bueno, Cáceres-

Rodríguez, \& Lima-

Rodríguez (2013)

$\mathrm{N}^{\circ}$ ítems
Formato
de
respuesta

9 ítems

Likert con 6 opciones:

TA-TD

35 ítems

Likert con 5 opciones: TA-TD.

\section{Contenido/Dimensiones/Componentes/ Escalas/Factores y Fiabilidad estimada}

Dos versiones: expresión de la sexualidad de

las mujeres y de los hombres.

Factores, fiabilidad y contenido: apertura sexual $(\alpha=.84)$ (acceso a la educación sexual y los métodos anticonceptivos, la libertad de expresión sexual, etc.) y tiempo (no fiabilidad).

Subescalas: percepción sexual, comportamientos sexuales, preservación de la virginidad de uno, doble moral sexual y pornografía.

Fiabilidad: $\alpha=.88$.

Actitud sexual positiva: sexualidad percibida

10 ítems

Likert con 5 opciones: TA-TD. como satisfactoria, alejada de mitos y tabúes, basada en el respeto a las personas de distinto género u orientación sexual, y con inclinación al uso de medidas de prevención. Fiabilidad: $\alpha=.73$.

\section{Objetivo y población explicitados}

Nota: TA-TD: totalmente de acuerdo-totalmente en desacuerdo. CR: coeficiente de reproductibilidad. CE: coeficiente de escalabilidad. $\alpha$ : alfa de Cronbach (coeficiente de consistencia interna). $r=$ correlación testretest (coeficiente de estabilidad).

Tabla 2. Revisión de instrumentos con alguna escala o subescala que mide actitudes hacia la sexualidad

\begin{tabular}{|c|c|c|c|}
\hline $\begin{array}{l}\text { Título } \\
\text { Autores y año de la } \\
\text { primera referencia }\end{array}$ & $\begin{array}{l}\mathrm{N}^{\circ} \text { ítems } \\
\text { Formato de } \\
\text { respuesta }\end{array}$ & $\begin{array}{l}\text { Contenido/Dimensiones/Componentes/ } \\
\text { Escalas/Factores y Fiabilidad estimada }\end{array}$ & $\begin{array}{l}\text { Objetivo y población } \\
\text { explicitados }\end{array}$ \\
\hline $\begin{array}{l}\text { 1.The Mosher Forced } \\
\text { Choice Sex Guilt Scale } \\
\text { (revision en } 1988 \text { y versión } \\
\text { breve en 2011) } \\
\text { Mosher (1961) }\end{array}$ & $\begin{array}{c}\text { Culpabilidad } \\
\text { sexual: } 50 \\
\text { ítems, } 10 \\
\text { ítems (2011) } \\
\text { Likert con } 7 \\
\text { opciones: } \\
\text { no es } \\
\text { absolutamente } \\
\text { cierto para mí- } \\
\text { muy cierto } \\
\text { para mí. }\end{array}$ & $\begin{array}{l}\text { Culpabilidad sexual: tendencia a sentirse } \\
\text { culpable por la violación de las normas acerca } \\
\text { de las conductas sexuales aceptables. } \\
\text { Fiabilidad: } \alpha \text { alrededor de } .90 . \\
\end{array}$ & $\begin{array}{l}\text { Nivel de culpabilidad sexual en } \\
\text { una amplia población. }\end{array}$ \\
\hline $\begin{array}{l}\text { 2. Sex, Knowledge and } \\
\text { Attitude Test (SKAT) } \\
\text { Lief \& Reed (1972) }\end{array}$ & $\begin{array}{l}35 \text { ítems } \\
\text { Likert con } 5 \\
\text { opciones: TA- } \\
\text { TD }\end{array}$ & $\begin{array}{l}\text { Subescalas y fiabilidad: relaciones sexuales } \\
\text { heterosexuales prematrimoniales y extrama- } \\
\text { trimoniales }(\alpha=.86) \text {, mitos sexuales }(\alpha=.81) \text {, } \\
\text { masturbación }(\alpha=.81) \text { y aborto }(\alpha=.80) \text {. }\end{array}$ & $\begin{array}{l}\text { Actitudes hacia la sexualidad en } \\
\text { profesionales de la salud. }\end{array}$ \\
\hline $\begin{array}{l}\text { 3. Sexual Knowledge and } \\
\text { Attitude Test for } \\
\text { Adolescents (SKAT-A) } \\
\text { (adaptación del SKAT y } \\
\text { revisión en 2005) } \\
\text { Lief, Fullard, \& Devlin(1990) }\end{array}$ & $\begin{array}{l}40 \text { ítems } \\
\text { Likert con } 5 \\
\text { opciones: TA- } \\
\text { TD. }\end{array}$ & $\begin{array}{l}\text { Factores: sexualidad prematrimonial, } \\
\text { violación/coerción sexual, homosexualidad, } \\
\text { pornografía, masturbación y aborto. } \\
\text { Fiabilidad: } \alpha=.88 \text { y } \mathrm{r}(\text { dos semanas })=.89 \\
\text { (universitarios); } \alpha=.82 \text { y } \mathrm{r}(\text { dos semanas })= \\
.89 \text { (estudiantes secundaria). }\end{array}$ & $\begin{array}{l}\text { Evaluación subjetiva con respecto } \\
\text { al comportamiento sexual y la } \\
\text { experiencia sexual en jóvenes } \\
\text { entre } 12 \text { y } 18 \text { años. }\end{array}$ \\
\hline
\end{tabular}


Tabla 2 (cont.). Revisión de instrumentos con alguna escala o subescala que mide actitudes hacia la sexualidad

\begin{tabular}{|c|c|c|c|}
\hline $\begin{array}{l}\text { Título } \\
\text { Autores y año de la } \\
\text { primera referencia }\end{array}$ & $\begin{array}{l}\mathrm{N}^{0} \text { ítems } \\
\text { Formato de }\end{array}$ & $\begin{array}{l}\text { Contenido/Dimensiones/Componentes/ } \\
\text { Escalas/Factores y Fiabilidad estimada }\end{array}$ & $\begin{array}{l}\text { Objetivo y población } \\
\text { explicitados }\end{array}$ \\
\hline
\end{tabular}

4. Self-Image

Questionnaire

Offer (1972)

5. Derogatis Sexual Functioning Inventory (DSFI)

Derogatis (1975)

6. Sexual Attitude and Information Questionnaire (SAIQ)

Brockway et al. (1978)

7. Frenken Sexual

Experience Scales

Frenken \& Vennix (1978)

8. Sexual knowledge and attitudes towards sexuality

Lewin \& Helmius (1983)

9. Mathtech Attitude and Value (MAV) Scale

Kirby (1984)

10. Sexuality Survey of Nurses Caring for Cancer Patients

Williams, Wilson, Hongladarom, \& McDonell (1986)

11. Sexuality Survey of

Nurses Caring for

Adolescents (basada en la

Sexuality Survey of Nurses Caring for Cancer Patients)
10 ítems

Likert con 6 Actitudes sexuales: erotofobia-erotofilia y opciones: me autopercepción del atractivo hacia el otro describe muy género.

bien-no me Fiabilidad: $\alpha=.73$ (chicos y chicas de 12 a 16 describe en absoluto.

30 ítems $(15$

liberales y 15 Contenido: relaciones prematrimoniales y conservadores). extramatrimoniales, homosexualidad,

Likert con 5 opciones: TATD. masturbación, múltiples parejas sexuales y prácticas sexuales controvertidas.

Fiabilidad: $\alpha=.80$.

\section{4 ítems}

Likert con 6

opciones: TA- Escalas y fiabilidad: aceptabilidad de

TD/nada comportamiento sexual $(r=.91)$ y

preocupado- preocupaciones sexuales $(\mathrm{r}=.83)$. muy preocupado.

The Sexual

Morality

Scale: Likert con 5

opciones: TAno aceptada en absoluto/en ocasiones 6 opciones (sin experiencia).

12 ítems

Likert con 5 opciones: TATD.

\section{Contenido: comportamiento sexual}

matrimonial, sexo prematrimonial, comportamientos sexuales, socialización sexual de los niños y los jóvenes.

Subescalas: moralidad sexual dentro del matrimonio, experiencia sexual premarital y socialización sexual moral.

Factores y fiabilidad: búsqueda de novedad sexual $(\alpha=.71)$, individualización e igualdad ( $\alpha=.65)$ y fidelidad $(\alpha=.71)$.

Escalas y fiabilidad: actitud hacia la vida 25 items sexual $(\alpha=.75)$, actitud hacia la importancia del control de la natalidad $(\alpha=.72)$, actitud Likert con 5 hacia las relaciones prematrimoniales $(\alpha=$ opciones: TA- .94), actitud hacia usar la presión y la fuerza TD. en la actividad sexual $(\alpha=.68)$ y actitud hacia los roles de género $(\alpha=.66)$.

7 ítems

Likert con 6

opciones: TA-

TD.

\section{2 items}

Likert con 6 Contenido: preocupaciones sexuales, opciones: TA- asesoramiento sexual, educación sexual, etc. TD.
Actitudes sexuales principalmente en chicas adolescentes, con el objetivo de detectar distorsión de la imagen.

Actitudes sexuales conservadoras y liberales en población general con el objetivo de medir función sexual.

Grado de aceptación de comportamientos sexuales y el grado de preocupación en varias áreas relacionadas con la sexualidad, en personas con lesión de la medula espinal y sus parejas, tras un programa de asesoramiento y educación sexual.

Diversos aspectos de las actitudes hacia la sexualidad (moralidad permisiva-restrictiva) en una amplia población de mujeres (esclerosis múltiple, lesiones medulares, epilepsia, sin enfermedades, etc.).

Actitudes hacia la sexualidad en estudiantes mayores de 18 años.

Actitudes hacia determinados aspectos de la sexualidad en adolescentes y adultos jóvenes.

Actitudes hacia la sexualidad de las enfermeras en relación a sus pacientes con cáncer.

Actitudes liberales y conservadoras hacia la sexualidad de las enfermeras en relación a sus pacientes adolescentes. 
Tabla 2 (cont.). Revisión de instrumentos con alguna escala o subescala que mide actitudes hacia la sexualidad

\section{Título \\ Autores y año de la primera referencia \\ Contenido/Dimensiones/Componentes/ Escalas/Factores y Fiabilidad estimada}

12. Sociosexual

Orientation Inventory

(SOI) (revisión en 2008)

Simpson \& Gangestad (1991)

\section{Sociosexuality Scale} (SS)

Bailey, Kirk, Zhu, Dunne, \& Martin (2000)

14. Sexual Dysfunctional Beliefs Questionnaire (SDBQ)

Nobre, Pinto-Gouveia, \& Gomes (2003)

15. Sexual Activities and Attitude Questionnaire (SAAQ)

Noll, Trickett, \& Putnam (2003)

6. Cross-Cultural Attitude Scale (CCAS)

Leiblum, Wiegel, \&

Brickle (2003)

17. Sexuality Attitude and Beliefs Survey (SABS)

Reynolds \& Magnan (2005)

\section{Index Liberal Sexual} Attitude

Preston \& Brown-Hart (2006)

Fiabilidad: $\alpha=.75-.82 ; \mathrm{r}(7-10$ días $)=.85$.

19. Attitude toward sex and Likert con 6 perception on sexual opciones: muy relationship

Kim (2008)
12 ítems

Likert con 6 opciones: TA-

TD.

\section{4 ítems}

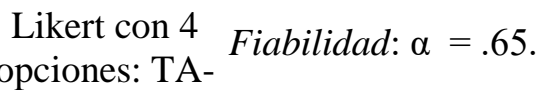

TD.

20 ítems

Contenido: actitud hacia el sexo sin amor y confort con sexo casual.

Likert con 9 Fiabilidad: $\alpha=.83$ y $\mathrm{r}$ (un año) $=.79$

TD. ${ }^{-}$(mujeres); $\alpha=.87$ y r (un año) $=.73$ (hombres).

20 ítems Contenido: ítems del SOI y del Eysenck's

Likert con 4 Inventory of Attitude to Sex.

TD. .88 .

Fiabilidad: $\alpha$ (mujeres $)=.85 ; \alpha$ (hombres $)=$

Sexual

Conservatism:

(mujeres)

(hombres). Dos versiones: hombre y mujer.

Likert con 5 Fiabilidad: $\alpha$ (mujeres) $=.81 ; \alpha$ (hombres $)=$ .93

de acuerdo-

completamente

en desacuerdo

Ordenador Factores y fiabilidad: permisividad sexual ( $\alpha$ computerizado: $=.96)$, preocupación sexual $(\alpha=.91)$, de los auriculares $=.70$ ).

y contestan con

53 ítems

Likert con 5 Áreas principales: roles de género, confort o TD.

de acuerdo-en

absoluto de

acuerdo.

\section{Objetivo y población explicitados}

Actitudes hacia el sexo sin compromiso (constructo sociosexualidad u orientación sociosexual) en población general.

Actitudes sexuales en una población amplia (hombres y mujeres, mujeres víctimas de violencia, mujeres con alto riesgo de victimización, etc.).

Creencias sexuales disfuncionales como un indicador de factores vulnerables en personas con trastornos sexuales (Portugal).
Aversión sexual (baja permisividad sexual) y la ambivalencia sexual en mujeres maltratadas y con trastornos de personalidad.

Actitudes sexuales conservadoras y liberales y búsqueda de comportamientos de cuidado de salud en profesionales sanitarios de diferentes culturas.

Actitudes y creencias hacia la sexualidad humana en relación con la práctica de la enfermería, con el objetivo de detectar las barreras que pueden afectar al asesoramiento sexual hacia los pacientes en enfermeras.

Actitud hacia diferentes tipos de comportamiento sexual en hombres (en prisión) clientes de prostitutas de la calle.

Actitud hacia el sexo y la percepción en las relaciones sexuales en hombres que consumen sildenafilo (problemas de erección). 
Tabla 2 (cont.). Revisión de instrumentos con alguna escala o subescala que mide actitudes hacia la sexualidad

\begin{tabular}{|c|c|c|c|}
\hline $\begin{array}{l}\text { Título } \\
\begin{array}{l}\text { Autores y año de la } \\
\text { primera referencia }\end{array}\end{array}$ & $\begin{array}{l}\mathrm{N}^{\circ} \text { ítems } \\
\text { Formato de } \\
\text { respuesta }\end{array}$ & $\begin{array}{l}\text { Contenido/Dimensiones/Componentes/ } \\
\text { Escalas/Factores y Fiabilidad estimada }\end{array}$ & $\begin{array}{l}\text { Objetivo y población } \\
\text { explicitados }\end{array}$ \\
\hline & 30 ítems & & \\
\hline $\begin{array}{l}\text { 20. Sexual Attitudes and } \\
\text { Experiences Survey } \\
\text { (SAES) } \\
\text { Tobin (2011) }\end{array}$ & $\begin{array}{l}\text { Likert con } 5 \\
\text { opciones: } \\
\text { aprobar } \\
\text { fuertemente- } \\
\text { desaprobar } \\
\text { fuertemente. }\end{array}$ & Fiabilidad: $\alpha=.90$ & $\begin{array}{l}\text { Actitudes sexuales con el } \\
\text { objetivo de relacionarlas con la } \\
\text { experiencia sexual en estudiantes. }\end{array}$ \\
\hline
\end{tabular}

Nota: TA-TD: totalmente de acuerdo-totalmente en desacuerdo. $\alpha$ : alfa de Cronbach (coeficiente de consistencia interna). $r=$ correlación test-retest (coeficiente de estabilidad).

Tabla 3. Revisión de instrumentos que miden las actitudes sexuales hacia un colectivo específico

\begin{tabular}{|c|c|c|c|c|}
\hline $\begin{array}{l}\text { Título } \\
\text { Autores y año de la } \\
\text { primera referencia }\end{array}$ & $\begin{array}{l}\mathrm{N}^{0} \text { ítems } \\
\text { Formato de } \\
\text { respuesta }\end{array}$ & $\begin{array}{l}\text { Contenido/Dimensiones/Componentes } \\
\text { /Escalas/ } \\
\text { Factores y Fiabilidad estimada }\end{array}$ & $\begin{array}{c}\text { Población } \\
\text { objetivo }\end{array}$ & $\begin{array}{c}\text { Objetivo } \\
\text { explicitado }\end{array}$ \\
\hline $\begin{array}{l}\text { 1.Aging Sexual Knowledge } \\
\text { and Attitude Scale } \\
\text { (ASKAS) } \\
\text { White (1982) }\end{array}$ & $\begin{array}{l}\text { Escala de } \\
\text { Actitudes: } 26 \\
\text { ítems } \\
\text { Likert con } 7 \\
\text { opciones: } \\
\text { acuerdo- } \\
\text { desacuerdo. }\end{array}$ & $\begin{array}{l}\text { Fiabilidad: } \alpha \text { (personas mayores })=.87 ; \\
\alpha \text { (trabajadores con personas mayores })= \\
.85 ; \alpha(\text { familiares personas mayores })= \\
.86 .\end{array}$ & $\begin{array}{l}\text { Personas mayores, } \\
\text { personas que } \\
\text { trabajan con } \\
\text { personas mayores } \\
\text { y miembros de la } \\
\text { familia de } \\
\text { personas mayores. }\end{array}$ & $\begin{array}{l}\text { Actitudes hacia el } \\
\text { comportamiento } \\
\text { sexual (permisividad } \\
\text { sexual) de personas } \\
\text { mayores. }\end{array}$ \\
\hline $\begin{array}{l}\text { 2. Questionnaire about } \\
\text { attitudes of staff in services } \\
\text { for people with intellectual } \\
\text { disabilities } \\
\text { Ryan \& McConkey (2000) }\end{array}$ & $\begin{array}{l}10 \text { ítems } \\
\text { Likert con } 5 \\
\text { opciones: } \\
\text { completamente } \\
\text { de acuerdo- } \\
\text { completamente } \\
\text { en desacuerdo. }\end{array}$ & $\begin{array}{l}\text { Factores: educación sexual, expresión } \\
\text { adulta de la sexualidad, no tener hijos y } \\
\text { actitudes conservadoras. }\end{array}$ & $\begin{array}{l}\text { Profesionales que } \\
\text { trabajan con } \\
\text { personas } \\
\text { discapacitadas } \\
\text { (Irlanda). }\end{array}$ & $\begin{array}{l}\text { Actitudes sexuales } \\
\text { hacia las personas } \\
\text { discapacitadas. }\end{array}$ \\
\hline $\begin{array}{l}\text { 3. Greek Sexuality } \\
\text { Attitudes Questionnaire-- } \\
\text { Learning Disabilities } \\
\text { (GSAQ-LD) } \\
\text { Karellou (2003) }\end{array}$ & $\begin{array}{l}45 \text { ítems } \\
\text { Likert con } 5 \\
\text { opciones. }\end{array}$ & $\begin{array}{l}\text { Escalas y fiabilidad: sexualidad humana } \\
(\mathrm{r}=.80) \text {, reconocimiento }(\mathrm{r}=.64) \text {, } \\
\text { discriminación }(\mathrm{r}=.72) \mathrm{y} \\
\text { homosexualidad }(\mathrm{r}=.54) .\end{array}$ & $\begin{array}{l}\text { Población general } \\
\text { (Grecia). }\end{array}$ & $\begin{array}{l}\text { Actitudes hacia la } \\
\text { sexualidad de las } \\
\text { personas con y sin } \\
\text { discapacidad } \\
\text { intelectual. }\end{array}$ \\
\hline $\begin{array}{l}\text { 4. Attitudes to Sexuality } \\
\text { Questionnaire (Individuals } \\
\text { with an Intellectual } \\
\text { Disability) (ASQ-ID) } \\
\text { (revision 2007) } \\
\text { Cuskelly \& Bryde (2004) }\end{array}$ & $\begin{array}{l}33 \text { ítems } \\
\text { Likert con } 6 \\
\text { opciones: TA- } \\
\text { TD. }\end{array}$ & $\begin{array}{l}\text { Contenido: sentimientos sexuales, } \\
\text { educación sexual, masturbación, } \\
\text { relaciones personales, relaciones } \\
\text { sexuales, esterilización, matrimonio y } \\
\text { parentalidad. } \\
\text { Factores y fiabilidad: derechos sexuales } \\
\text { ( } \alpha=.93) \text {, crianza }(\alpha=.88), \\
\text { comportamiento sexual no reproductivo } \\
(\alpha=.84) \text { y autocontrol }(\alpha=.67) \text {. }\end{array}$ & $\begin{array}{l}\text { Profesionales que } \\
\text { trabajan o } \\
\text { interactúan con } \\
\text { discapacitados } \\
\text { intelectuales } \\
\text { (Australia). }\end{array}$ & $\begin{array}{l}\text { Actitudes hacia la } \\
\text { sexualidad de } \\
\text { adultos con } \\
\text { discapacidad } \\
\text { intelectual. }\end{array}$ \\
\hline $\begin{array}{l}\text { 5. Escala de Actitudes } \\
\text { hacia la Sexualidad en el } \\
\text { Envejecido }\end{array}$ & $\begin{array}{l}44 \text { ítems } \\
\text { Likert con } 4 \\
\text { opciones. }\end{array}$ & $\begin{array}{l}\text { Factores: estereotipos sexuales, mitos } \\
\text { sociales/religiosos, prácticas sexuales, } \\
\text { deseo sexual y rol de la mujer en el } \\
\text { sexo. } \\
\text { Fiabilidad: } \alpha=.95 \text {. }\end{array}$ & $\begin{array}{l}\text { Personas mayores } \\
\text { (Puerto Rico). }\end{array}$ & $\begin{array}{l}\text { Actitudes hacia la } \\
\text { sexualidad en } \\
\text { personas mayores. }\end{array}$ \\
\hline
\end{tabular}

Nota: TA-TD: totalmente de acuerdo-totalmente en desacuerdo. $\alpha$ : alfa de Cronbach (coeficiente de consistencia interna). $r=$ correlación test-retest (coeficiente de estabilidad). 


\section{Referencias}

Beere, C. A. (1990). Sex and gender issues. A handbook of tests and measures. Westport, CT: Greenwood.

Breckler, S. J. (1984). Empirical validation of affect, behavior, and cognition as distinct components of attitude. Journal of Personality and Social Psychology, 47, 1191-1205. doi: 10.1037/0033-2909.107.2.260

Brockway, J. A., Steger, J. C., Berni, R., Ost, V. V., Williamson-Kirkland, T. E., \& Peck, C. L. (1978). Effectiveness of a sex education and counseling program for spinal cord injured patients. Sexuality and Disability, 1, 127-136. doi: 10.1007/BF01101773

Brown, R. (1995). Prejuicio. Su Psicología Social. Madrid: Alianza Editorial.

Croake, J. W., \& James, B. (1973). A four year comparison of premarital sexual attitudes. Journal of Sex Research, 9, 91-96. doi: 10.1080/00224497309550784

Cunningham, W. A., Zelazo, P. D., Packer, D. J., \& Van Bavel, J. J. (2007). The iterative reprocessing model: A multilevel framework for attitudes and evaluation. Social Cognition, 25, 736-760. doi: 10.1521/soco.2007.25.5.736

Cuskelly, M., \& Gilmore, L. (2007). Attitudes to Sexuality Questionnaire (Individuals with an Intellectual Disability): Scale development and community norms. Journal of Intellectual \& Developmental Disability, 32, 214-221. doi. 10.1080/13668250701549450

Eagly, A. H., \& Chaiken, S. (1993). The psychology of attitudes. Fort Worth, TX: Harcourt, Brace, Jovanovich.

Eysenck, H. J. (1970). Personality and attitudes to sex: A factorial study. Personality, 1, 355376.

Fabrigar, L. R., MacDonald, T. K., \& Wegener, D. T. (2005). The structure of attitudes. In D. Albarracin, B. T. Johnson, \& M. P. Zanna (Eds.), Handbook of attitude and attitude change (pp. 79-124). Mahwah, NJ; Erlbaum.

Fisher, W. A., Byrne, D., White, L. A., \& Kelley, K. (1988). Erotophopia-erotophilia as a dimension of personality. Journal of Sex Research, 25, 123-151. doi: 10.1080/0022449 8809551448
Fisher, T. D., Davis, C. M., Yarber, W. L., \& Davis, S. L. (2011). Handbook of sexualityrelated measures. New York: Routledge.

Glenn, N. D., \& Weaver, C. N. (1979). Attitudes toward premarital, extramarital, and homosexual relations in the U.S. in the 1970s. Journal of Sex Research, 15, 108-118. doi: 10.1080/00224497909551029

Gray, P. B. (2013). Evolution and human sexuality. American Journal of Physical Anthropology, 152 (Suppl 57), 94-118. doi: 10.1002/ajpa.22394.

Hendrick, S., Hendrick C., Slapion-Foote, M. J., \& Foote, F. H. (1985). Gender differences in sexual attitudes. Journal of Personality and Social Psychology, 48, 1630-1642. doi: 10.1037/0022-3514.48.6.1630

Hendrick, C., Hendrick, S. S., \& Reich, D. A. (2006). The brief sexual attitudes scale. Journal of Sex Research, 43, 76-86. doi: 10.1080/00224490609552301

Hudson, W. W., Murphy, G. J., \& Nurius, P. S. (1983). A short-form scale to measure liberal versus conservative orientations toward human sexual expression. Journal of Sex Research, 19, 258-272. doi: 10.1080/00224498309551186

Lefkowitz, E. S., Gillen, M. M., Shearer, C. L., \& Boone, T. L. (2004). Religiosity, sexual behavior, and sexual attitudes during emerging adulthood. Journal of Sex Research, 41, 150-159. doi: 10.1080/002244904095522 23

Lief, H. I., Fullard, W., \& Devlin, J. (1990). A new measure of adolescent sexuality: SKATA. Journal of Sex Education and Therapy, 16, 79-91. doi: 10.1080/01614576.1990.11074980

Lief, H. I., \& Reed, D. M. (1972). Sex Knowledge and Attitude Test. Philadelphia: University of Pennsylvania, Center for the Study of Sex Education in Medicine.

López, F. (2009). La Educación Sexual (2ºd.). Madrid: Biblioteca Nueva.

Lottes, I. L., \& Kuriloff, P. J. (1994). Sexual socialization differences by gender, Greek membership, ethnicity, and religious background. Psychology of Women Quarterly, 18 , 203-219. doi: 10.1111/j.14716402.1994.tb00451.x 
Lozano, I., \& Díaz-Loving, R. (2010). Medición de la homofobia en México: Desarrollo y validación. Revista Iberoamericana de Diagnóstico y Evaluación - e Avaliação Psicológica, 30, 105-124.

Luquis R. R., Brelsford, G. M., \& Pérez, M. A. (2015). Exploring latino college students' sexual behaviors in relation to their sexual attitudes, religiousness, and spirituality. Journal of Religion and Health, 54, 13451357. doi: 10.1007/s10943-014-99299

Luquis, R. R., Brelsford, G. M., \& Rojas-Guyler, L. (2012). Religiosity, spirituality, sexual attitudes, and sexual behaviors among college students. Journal of Religion and Health, 51, 601-614. doi: 10.1007/s10943-011-9527-z

Marks, M. J., \& Fraley, R. C. (2005). The sexual double standard: Fact or fiction? Sex Roles, 52, 175-186. doi: 10.1007/s11199-005-1293-5

Martínez, I., Reyes, I., \& Paredes, R.G. (2010). Autoesquema sexual femenino: Construcción $\mathrm{y}$ validación de una escala para población mexicana. Revista Iberoamericana de Diagnóstico y Evaluación - e Avaliação Psicológica, 30, 143-155.

McMillen, E. K., Helm Jr, H. W., \& McBride, D. C. (2011). Religious orientation and sexual attitudes and behaviors. Journal of Research on Christian Education, 20, 195-206. doi: 10.1080/10656219.2011.590755

Muehlenhard, C. L., \& Quackenbush, D. M. (1996). The social meaning of women's condom use: The sexual double standard and women's beliefs about the meaning ascribed to condom use. Manuscrito no publicado.

Nobre, P. J., Pinto-Gouveia, J., \& Gomes, F. A. (2003). Sexual Dysfunctional Beliefs questionnaire: An instrument to assess sexual dysfunctional beliefs as vulnerability factors to sexual problems. Sexual and Relationship Therapy, 18, 171-204. doi: 10.1080/1468199031000061281

Offer, D. (1972). Attitudes toward sexuality in a group of 1500 middle class teen-agers. Journal of Youth and Adolescence, 1, 81-90. doi: 10.1007/BF01537065

Organización Mundial de la Salud (OMS). (2006). Defining sexual health Report of a technical consultation on sexual health 28-31 January 2002. Ginebra: OMS.

Redfearn, A. A., \& Laner, M. R. (2000). The effects of sexual assault on sexual attitudes. Marriage \& Family Review, 30, 109-125. doi: 10.1300/J002v30n01_08

Reiss, I. L. (1964).The Scaling of Premarital Sexual Permissiveness. Journal of Marriage and Family, 26, 188-198.

Reiss, I. L. (1989). Is this my scale? Journal of Marriage and Family, 51, 1079-1080.

Reiss, I. L. (2006). An insider's view of sexual science since Kinsey. Lanham, MD: Rowman $\&$ Littlefield.

Schofield, M. (1965). The sexual behavior of young people. Boston, MA: Little \& Brown.

Sprecher, S., \& Hatfield, E. (1996). Premarital sexual standards among U.S. college students: Comparisons with russian and japanese students. Archives of Sexual Behavior, 25, 261-288. doi: 10.1007/BF02438165

Sprecher, S., McKinney, K., Walsh, R., \& Anderson, C. (1988). "A revision of the Reiss Premarital Sexual Permissiveness Scale". Journal of Marriage and the Family, 50, 821828. doi: $10.2307 / 352650$

Thorne, F. (1966). The sex inventory. Journal of Clinical Psychology, 22, 367-374. doi: 10.1002/1097-4679(196610)

Trueblood, K., Hannon, R., \& Hall, D. S. (1998, Junio). Development and validation of a measure of sexual attitudes. Paper presented at the meeting of the Society for the Scientific Study of Sexuality Western Region Annual Conference, Honolulu, HI.

Weis, D. L., \& Felton, J. R. (1987). Marital exclusivity and the potential for future marital conflict. Social Work, 32, 45-49. doi: 10.1093/sw/32.1.45

Wells, B. E., \& Twenge, J. M. (2005). Changes in young people's sexual behavior and attitudes, 1943-1999: A cross-temporal meta-analysis. Review of General Psychology, 9, 249-261. doi: 10.1037/1089-2680.9.3.249

Zanna, M. P., \& Rempel, J. K. (1988). Attitudes: A new look at an old concept. In D. Bar-Tal \& A. W. Kruglanski (Eds.), The social psychology of knowledge (pp.315-334). Cambridge, England; Cambridge University Press.

Revista Iberoamericana de Diagnóstico y Evaluación - e Avaliação Psicológica. RIDEP · No43 · Vol.1 · 17-32 · 2017 